\title{
Artificial Intelligence Based Drug Discovery Techniques for COVID-19 Detection
}

\author{
Kawal Arora ${ }^{1}$, Ankur Singh Bist ${ }^{2}$ \\ Signy Advanced Technologies, India \\ Address : Level 39, One Canada Square, Canary Wharf, London E14 5AB \\ e-mail: kawal@signy.io ${ }^{1}$, ankur@signy.io ${ }^{2}$
}

(APA style, Justify, Arial 10pt) Example:

To cite this document:

Arora, K., Bist, AS. (2020). Artificial Intelligence Based Drug Discovery Techniques for COVID-19 Detection. Aptisi Transactions On Technopreneurship (ATT), 2(2), 120-126.

DOI : https://doi.org/10.34306/att.v2i2.88

\begin{abstract}
Recent advancements in the area of drug discovery using artificial intelligence made it possible to speed up the hunt for new pharmaceuticals. Drugs like arbidol, atazanavir, ramdev sivir \& favipiravir are under testing phase to cure COVID-19. In this paper, we present systematic study of Al based drug discovery techniques suitable for COVID-19 detection.
\end{abstract}

Keywords: Coronavirus, artificial intelligence, drug discovery and drug selection.

\section{Introduction}

Coronavirus is a specific type of virus and as per inherited property replicates and expands. Seriousness of this virus is due to Its nature of expanding with very fast pace. Picture 1 shows the basic structure of coronavirus. Mammals and birds are attacking points of this virus. This disease cause variety of infections related to respiratory system.

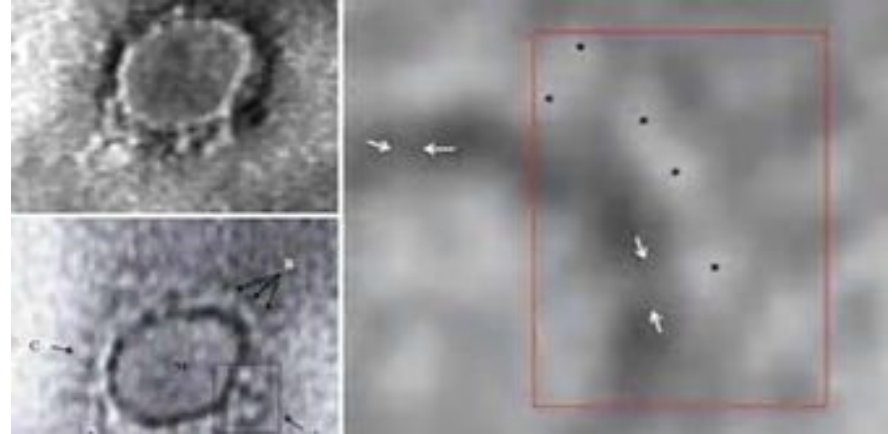

Picture 1: COVID-19 by electron microscope (Indian Council of Medical Research)

Coronavirus origination started from china and expanded in different parts of world. Most of the countries declared it as medical emergency. Till March 2020 around 40000 deaths are confirmed due to this. Picture 2 depicts the coronavirus spread. 


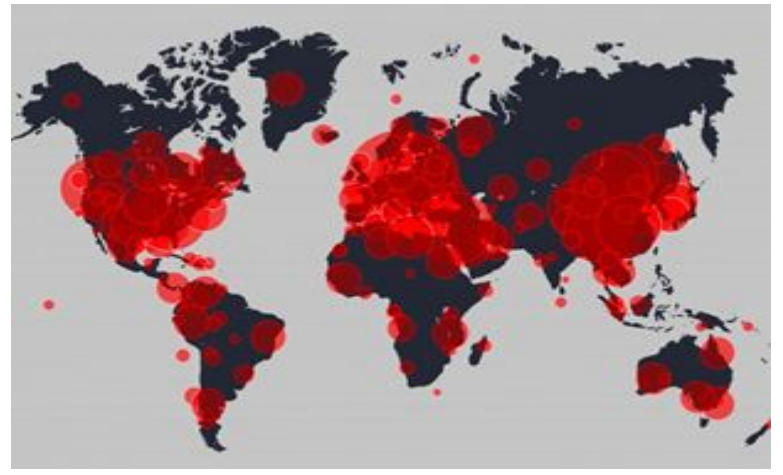

Picture 2: Coronavirus spread

Governments developed various isolation centers to take care infected patients. Sanitization procedures are taken at wide scale to mitigate the effect of this epidemic. Picture 3 shows the efforts made by government agencies to tackle the situation.

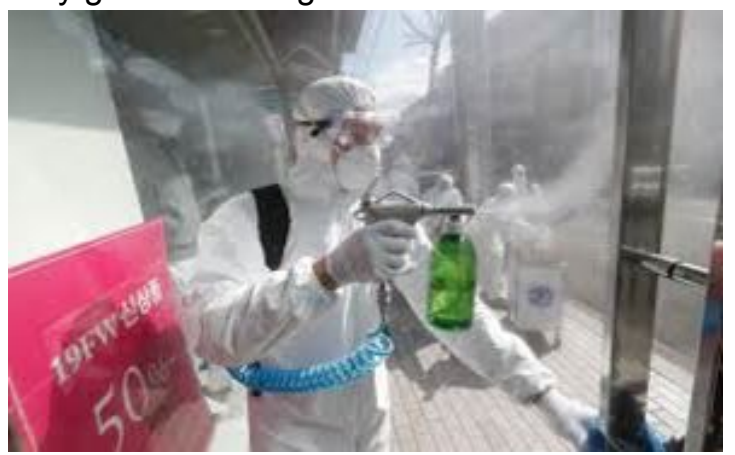

Picture 3: Coronavirus Prevention

Government efforts are not sufficient to tackle the situation alone. Without efforts of common men it's not possible to control the disaster. Picture 4 shows some common precautions required by everyone in this situation. In case of any symptoms, medical checkup is necessary. Common questions \& answers related to COVID-19 are released by WHO: https://www.who.int/news-room/q-a-detail/q-a-coronaviruses.

Advice for common public released by WHO can be found at: https://www.who.int/emergencies/diseases/novel-coronavirus-2019/advice-for-public.

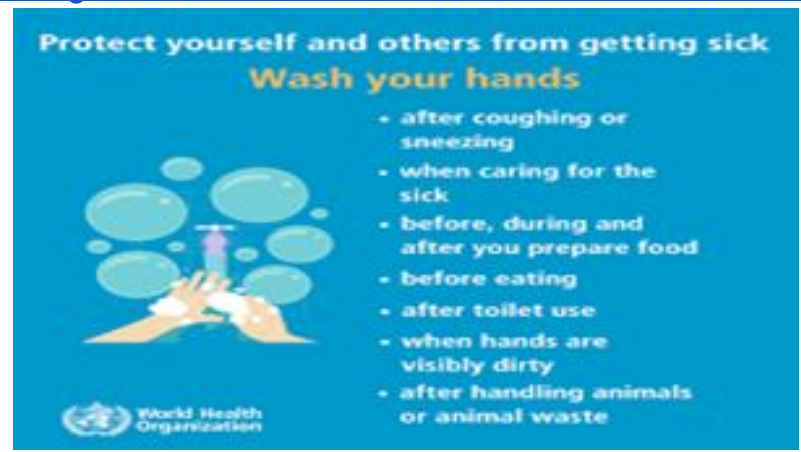

Picture 4: Preventive Measures

Al can be used to tackle this situation:

1. By analyzing Chest X-Ray, CT-Scans and other clinical symptoms to detect COVID-19

2. By doing drug or vaccine discovery for Covid-19 virus 
3. By analyzing the effect of existing medicines on Covid-19 virus

4. By analyzing the remedies for current and future attacks of same type

\section{Drug Discovery Based on Al}

Drug discovery is the process of finding drug for new diseases. It involves target identification, target validation, lead identification and lead optimization. Identification of protein that has specific function with disease is called target identification. Verification of target as per inventor thought process is called target validation. Process of identifying best compounds with respect to target protein is called lead identification. Lead optimization is the process of ensuring drug associated characteristics of compounds. Inventor has to assure about bioavailability, specificity and toxicity of identified compounds. The process of testing compounds with animals to check the response of compound is called pre-clinical testing. Picture 1 explains the drug discovery and development timeline.

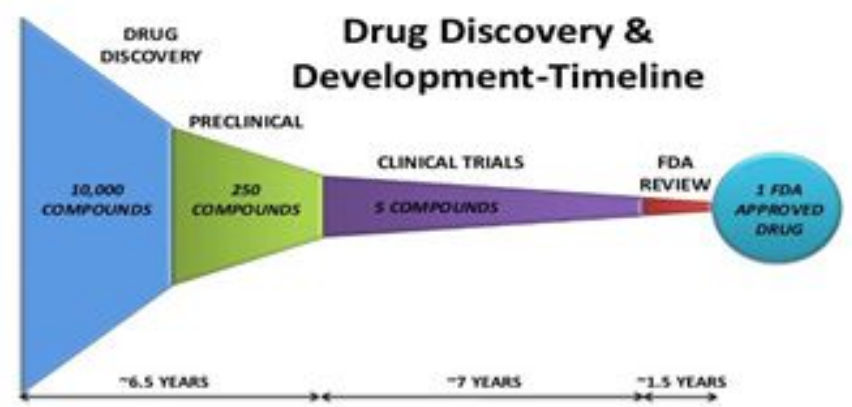

Picture 5: Drug discovery process [1]

COVID-19 is major issue in front of world; continuous process of drug/vaccine discovery is going by various research groups and pharma companies. As per research article $[2,3]$ antiviral drugs and process of treatment for COVID-19 is mentioned in table1.Still research is going on these drugs and actual medicine is yet to come.

\begin{tabular}{|c|c|c|}
\hline S.No. & Drug & Process of treatment \\
\hline 1 & IFN- $\alpha$ & $\begin{array}{c}\text { Vapor inhalation, 2 times/day, } \\
\text { duration of treatment: maximum 10 } \\
\text { days }\end{array}$ \\
\hline 2 & Lopinavir/ritonavir & $\begin{array}{c}\text { Oral, 2 times/day, duration of } \\
\text { treatment: maximum 10 days }\end{array}$ \\
\hline 3 & Ribavirin & $\begin{array}{c}\text { Intravenous infusion, 2-3 } \\
\text { times/day, duration of treatment: } \\
\text { maximum 10 days }\end{array}$ \\
\hline 4 & $\begin{array}{c}\text { Chloroquine } \\
\text { phosphate }\end{array}$ & $\begin{array}{c}\text { Oral, 2 times/day, duration of } \\
\text { treatment: maximum 10 days }\end{array}$ \\
\hline 5 & Arbidol & $\begin{array}{c}\text { Oral, 3 times/day, duration of } \\
\text { treatment: maximum 10 days }\end{array}$ \\
\hline
\end{tabular}

Table1: Treatment Guideline for COVID-19 [2,3]

As per research [3] following agents are found useful against SARS-CoV-2. In January 1996, Merck \& Co. found that indinavir was a clinically capable medicine depending on data from human trials. Saquinavir was found by the pharmaceutical company Roche and approved by food and drug administration, US. Similarly other drugs are verified by different agencies and worked well for other medical causes.

1. Indinavir

2. saquinavir

3. lopinavir 
4. carfilzomib

5. ritonavir

6. remdesivir

7. atazanavir

8. darunavir

9. tipranavir

10. fosamprenavir

11. Enzaplatovir

12. Presatovir

13. Abacavir

14. Bortezomib

15. Elvitegravir

16. Maribavir

17. Raltegravir

18. Montelukast

19. Deoxyrhapontin

20. Polydatin

21. chalcone

22. disulfiram

23. carmofur

24. shikonin

25. ebselen

26. tideglusib

27. PX- 12

28. TDZD-8

29. cyclosporin $A$

30. cinanserin

31. Rhizoma Polygoni Cuspidati

32. Radix Sophorae Tonkinensis

Al has been harnessed to develop compounds i.e. chemically correct and useful against new diseases. Al based algorithms can be trained with crucial characteristics of know drug. Trained Al program can organize new molecules based on learning and it will lead to the synthesis of useful compound. Al can be used in searching good compounds, drug targets, chemical modifications etc. Authors in paper [4] used Al techniques to speed up and optimize the drug selection process. MIT researchers used ML algorithm to find drug called halicin [5].

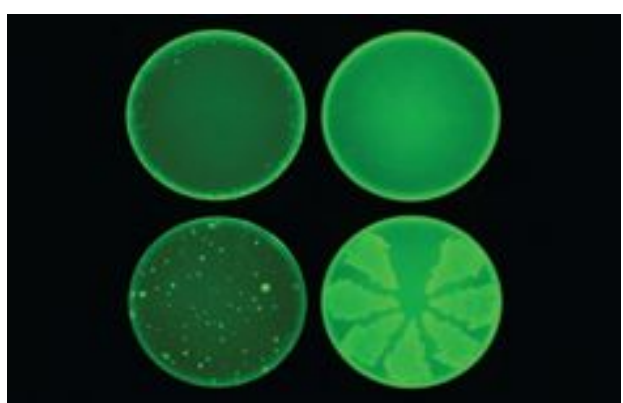

Picture 6: Halicin drug

Authors in paper $[6,7]$ discussed about Drug targeting and designing using artificial intelligence. Al subfields machine learning; deep learning and reinforcement learning are mainly used for sorting out various issues of healthcare. Let's tag Al subfields with healthcare related tasks under following points. 


\begin{tabular}{|c|c|c|}
\hline S.No. & Al Packages & Web links \\
\hline 1 & AlphaFold & https://github.com/deepmind/deepmind-research/tree/master/alph \\
afold_casp13
\end{tabular}

Table 2: Al for drug discovery

\subsection{Machine Learning}

1. Supervised Learning (Disease predication \& Drug efficacy)

2. Unsupervised Learning(Evaluating disease subtype \& disease target discovery)

\subsection{Deep Learning}

1. To process medical data for disease prediction like CT-Scan, Chest X-Ray etc.

\subsection{Deep Learning}

1. Drug design decision making

Researchers believe that the process of drug discovery will be totally changed with the advancements of artificial intelligence. 


\section{Al Based Tools And Techniques For Drug Discovery}

Moderna Therapeutics, a biotech company developed vaccine in context of COVID-19 in less than 2 months after the genetic sequence of the SARS-CoV-2 [7]. Researchers are using $\mathrm{Al}$ based technique to find molecule that can halt Coronavirus replication. Table2 contains summary of Al packages that can be used for drug discovery. AlphaFold, DeepChem, MOSES, Biopandas, Chemprop, OpenChem, ReLeaSE, Neural-fingerprint, HTMD, Gnina, ChemGAN challenge, DeepNeuralNet- QSAR, NNScore, SIEVE-Score, Hit Dexter are some Al based tools used for the task related to drug discovery, protein modeling etc. Let's discuss state of art Al systems for drug discovery. AlphaFold [9] is developed by DeepMind for protein folding prediction. AlphaFold pipeline use three different networks. At level1 variable auto-encoder (DRAW) is used to create fragments. At level2 simulated annealing is used to combine fragments then for last step, scoring process takes place with the help of co-evolutionary residues and scoring networks (3D based approach). Combination of protein model and energy model give rise to final protein. Picture 7 shows the protein structure prediction associated to COVID-19.

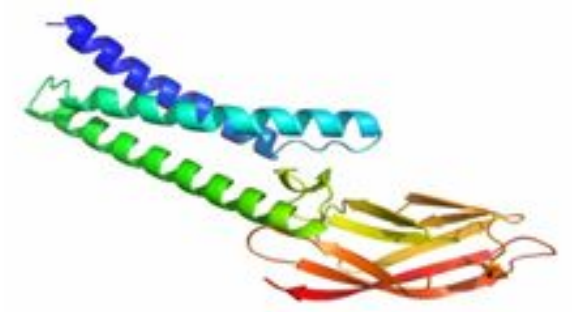

Picture 7: Protein structure prediction related to COVID-19[10]

DeepChem is open source tool that bring up the use of deep-learning in drug discovery. Drug discovery datasets are very costly and if available cannot be easily used due to ethical reasons. Author in experimental analysis [11] found that one-shot learning integrated with deepchem for drug discovery. Similarly other openly available tools as mentioned in table2 works on different aspect of drug discovery using artificial intelligence. Already various research communities and companies have used power of $\mathrm{Al}$ to find out drug suggestions for COVID-19. Clinical trials and approval process of drugs synthesized by Al for COVID-19 is going on. Everyone is hoping to get the outcome in this context as soon as possible.

\section{Conclusion And Future Work}

In this paper we summarized the current scenario of Al based drug discovery for COVID-19. Understanding protein synthesis, molecular changes, time management in laboratory are some important requirements to accelerate the process of drug discovery. $\mathrm{Al} / \mathrm{ML} / \mathrm{DL}$ techniques will work on each attribute of speedup drug discovery. Al based architectures are evolving with very fast pace, that will be helpful for designing robust system. Current work will be very useful for industrial or academic purpose.

\section{Acknowledgement}

This project is fully funded by Signy Advanced Technologies, Level 39 One Canada Square, Canary Wharf, London E14 5AB. 


\section{References}

[1]Raghavendra, M. S., John Rudolph Raj, and Arumugam Seetharaman. "A STUDY OF DECREASE IN R\&D SPENDING IN THE PHARMACEUTICAL INDUSTRY DURING POST-RECESSION." International Journal of Academic Research 4.5 (2012).

[2]Dong, Liying, Shasha $\mathrm{Hu}$, and Jianjun Gao. "Discovering drugs to treat coronavirus disease 2019 (COVID-19)." Drug Discoveries \& Therapeutics 14.1 (2020): 58-60.

[3]Guidelines for the Prevention, Diagnosis, and Treatment of Novel Coronavirus-induced Pneumonia, The 6th ed.

http://www.nhc.gov.cn/yzygj/s7653p/202002/8334a8326dd94d329df351d7da8aefc2/files/b21 8cfeb1bc54639af227f9 22bf6b817.pdf (accessed March 23, 2020). (in Chinese).

[4]Álvarez-Machancoses, Óscar, and Juan Luis Fernández-Martínez. "Using artificial intelligence methods to speed up drug discovery." Expert opinion on drug discovery 14.8 (2019): 769-777.

[5]http://news.mit.edu/2020/artificial-intelligence-identifies-new-antibiotic-0220, Last visited: 31 March 2020.

[6]Emig, D. et al. (2013) Drug target prediction and repositioning using an integrated network-based approach. PLoS One 8, e60618

[7]Duch, W. et al. (2007) Artificial intelligence approaches for rational drug design and discovery. Curr. Pharm. Des. 13, 1497-1508.

[8]https://theguardian.com/world/2020/mar/31/coronavirus-vaccine-when-will-it-be-ready, Last visited 31 March 2020.

[9]https://deepmind.com/blog/article/AlphaFold-Using-Al-for-scientific-discovery,Last visited: 31 March 2020.

[10]https://deepmind.com/research/open-source/computational-predictions-of-protein-structur es-associated-with-COVID-19, Last visited: 31 March 2020.

[11]https://www.microway.com/hpc-tech-tips/one-shot-learning-methods-applied-drug-discove ry-deepchem/, Last visited: 1 April 2020. 